\title{
The impact of tumor invasion to muscularis mucosae- vascular plexus on patient outcome in pT1 bladder urothelial carcinoma
}

\author{
Ahmet Sahan ${ }^{1}$, Fatma Gerin ${ }^{2}$, Asgar Garayev ${ }^{3}$, Emine Bozkurtlar ${ }^{2}$, Alkan Cubuk ${ }^{1}$, Orkunt Ozkaptan ${ }^{1}$, \\ Kasım Ertas ${ }^{4}$, Ylloren Tanidir ${ }^{3}$, Haydar Kamil Cam ${ }^{3}$, Ilker Tinay ${ }^{3}$ \\ ${ }^{1}$ Kartal Dr. Lutfi Kirdar Training and Research Hospital, Department of Urology, Istanbul, Turkey; \\ ${ }^{2}$ Marmara University, Department of Pathology, Istanbul, Turkey; \\ ${ }^{3}$ Marmara University, Department of Urology, Istanbul, Turkey; \\ ${ }^{4}$ Yuzuncu Yll University, Department of Urology, Van, Turkey.
}

\begin{abstract}
Summary Objectives: T1 bladder cancer has a wide range of tumor behavior and lamina propria invasion depth has a high potential risk of disease progression. To evaluate the patient outcome according to the tumor invasion to the muscularis mucosae-vascular plexus (MM-VP) in pT1 bladder urothelial carcinoma (BUC).

Materials and methods: This study is a retrospective analysis of patients consecutively recorded from 2007 to 2013. A total of 93 patients with a history of primary pT1 BUC and complete follow-up were included. We used a pathological substaging system according to the tumor invasion regarding the MM-VP: pTla (invasion above MM-VP) and pT1b (MM-VP invasion). We evaluated recurrence-free survival (RFS), progression-free survival (PFS), disease-specific-survival (DSS) based on this sub-staging system.

Results: Pathological evaluation regarding the MM-VP invasion revealed 53 patients (57\%) as pT1a BUC and 40 patients (43\%) as pT1b BUC. The mean follow-up was 78.8 months. During the follow-up period; 60 patients (64.5\%) had tumor recurrences, 32 patients (34.4\%) had progression to invasive disease, 18 patients (19.4\%) died during follow-up related to the BUC. In 29 (54.7\%) of pT1a and in 31(77.5\%) of pT1b tumors, the recurrent disease was recorded during the followup period ( $p=0.023$ ). DSS rates at 5 years for $p T 1 a$ and $p T 1 b$ were $80.2 \%$ and $60.8 \%$, respectively. PFS, RFS, and DSS rates were similar for $\mathrm{pT} 1 \mathrm{a} / \mathrm{pT} 1 \mathrm{~b}$ and did not reach statistical significance $(p>0.05)$.

Conclusions: Sub-staging of pT1 BUC according to the MM-VP invasion showed a limited impact on the outcome in our patient cohort. However, the presence of $\mathrm{pT} 1 \mathrm{~b}$ disease caused a significantly higher rate of recurrence.
\end{abstract}

KEY WORDS: T1 bladder cancer; Survival; Sub staging; Muscularis mucosae; Lamina propria depth.

Submitted 2 March 2020; Accepted 15 March 2020

\section{INTRODUCTION}

Bladder urothelial carcinoma (BUC) with pathological T1 stage represents a complex clinical dilemma due to its high rate of recurrence and progression. In fact, Tl BUC comprises a wide spectrum of different cases causing a huge clinical variability. At presentation, $75 \%$ of cases are non-muscle invasive bladder cancer (NMIBC), and approximately $70 \%$ of patients present as pTa, $20 \%$ as pT1, and $10 \%$ with carcinoma in situ (CIS) lesions (1).
In T1 high-grade tumors, 1 and 5 years of disease-progression rates are $11.4 \%$ and $19.8 \%$, respectively, and recurrence rates are between $21 \%$ to $53 \%$ despite intravesical treatments (2-7). This wide range in the recurrence and progression rates indicates the immense clinical variation of $\mathrm{pTl}$ patients. Therefore, the overall management of $\mathrm{Tl}$ tumors is a challenge for urologists. How to differentiate the clinically aggressive pTl tumors and how to provide the appropriate treatment strategy i.e. early cystectomy remains a difficult issue.

Tumor grade, stage, size of the tumor, multiplicity, and presence of CIS are known risk factors for recurrence and progression (1). Pathologic features like tumor growth pattern (papillary vs solid), tumor invasion pattern (broad vs trabecular vs infiltrative vs nested), and lymphovascular invasion were investigated to identify the variability of tumor behavior $(3,8,9)$. Factors dependent on surgery i.e. re-TUR has shown the benefit of recurrence and progression-free survival (10). However, no strict criteria to predict prognosis in $\mathrm{pTl}$ BUC have not been defined.

Due to a wide range of tumor behavior, there were many studies related to subclassification/sub-staging of T1 BUC since 1990 (11). These sub-staging systems were made according to the invasion of the muscularis mucosae-vascular plexus and invasion depth to lamina propria (11-13). Sub-staging according to MM-VP has been reported to be more superior than the invasion depth to lamina propria (14). However, the World Health Organization (WHO) (2004)/International Society of Urological Pathology (ISUP) and clinical guidelines do not recommend the sub-staging of $\mathrm{T} 1$ bladder cancer for the current daily practice $(15,16)$. Some reports showed that sub-staging is useful and predictive for progression of pT1 BUC $(2,12,17,18)$.

On the other hand, sub-staging is technically difficult and so far does not yield a clear prognostically significant separation on Tl bladder tumor $(19,20)$.

Therefore, new clinical series are required to evaluate the clinical utility of sub-staging in pTl BUC.

In the present study, we evaluated the impact of the invasion of the muscularis mucosae-vascular plexus (MMVP) on the clinical outcome of T1 BUC. 


\section{Patients ANd Methods}

We evaluated a total of 140 patients, who were referred to our center or diagnosed in our center with primary and pathologically reported pTl BUC between 2007 to 2013. Data of the patients were recorded prospectively in our electronic database and reviewed retrospectively for the study. The study protocol was approved by the local ethics committee (Number: 27-2014). Due to the retrospective nature of the study, only written consent was obtained from the patients.

The original pathology slides of all pTl bladder tumors were re-evaluated by two uropathologists $(\mathrm{EB} / \mathrm{FG})$ for stage and grade. We used the World Health Organization 2004 classifications to review grade. After this re-evaluation, initial pathological staging was confirmed in $82 \%$ of patients. We further excluded patients with a history of previous bladder cancer diagnosis, an absence of muscular layer, an absence of a repeated transurethral resection (Re-TUR) after 3 to 6 weeks, the presence of concomitant CIS and with upstaged/downstaged tumors based on this pathological re-evaluation. A total of 14 patients were lost to follow-up and as a result, 93 patients were eligible for final analysis. All patients received intravesical BCG treatment with at least 1 year duration. The follow-up cystoscopies and imaging of the upper urinary tract are planned according to the recommendation of EAU 15. Recurrences were defined as $\mathrm{pTa}, \mathrm{pTl}$ and CIS tumors and progression was defined as pT2 or higher stage and/or development of metastasis. We used a pathological sub-staging system

\section{Figure 1.}

Microscopic apperarnece.

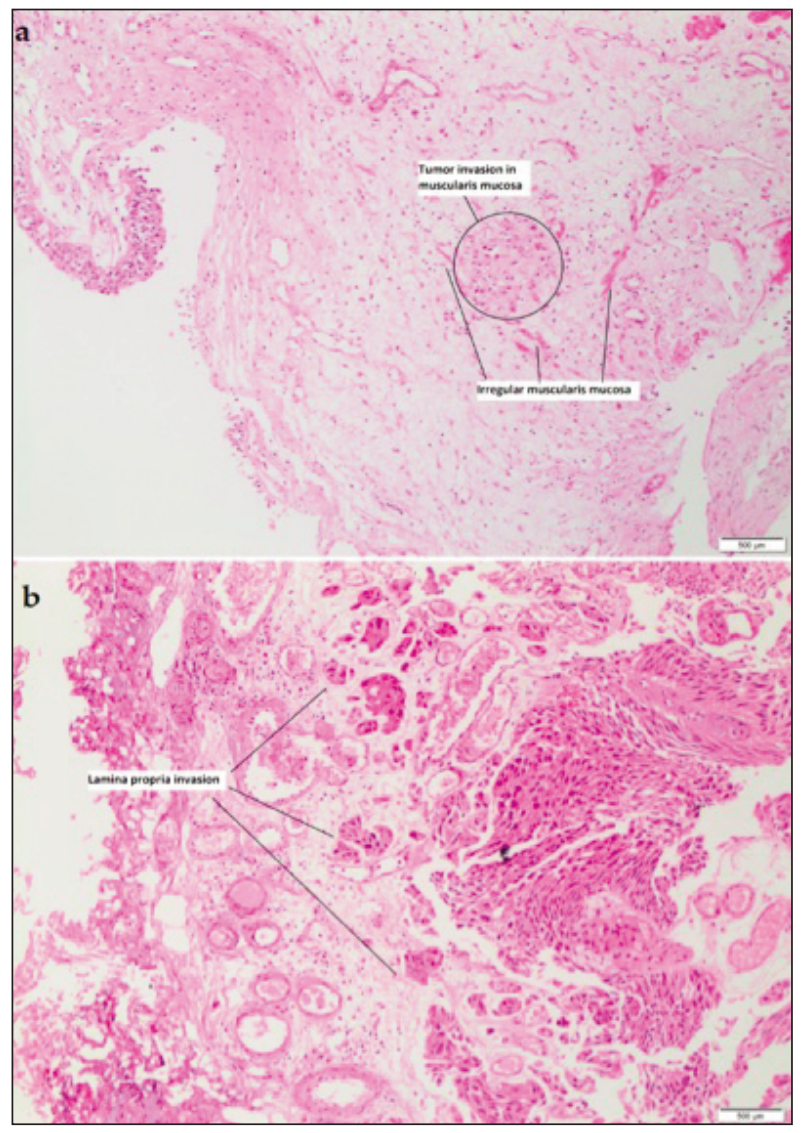

Table 1.

Characteristics of the patients based on the two sub-staging systems.

\begin{tabular}{|c|c|c|c|c|}
\hline & & T1a (n: 53) & T1b (n: 40) & $P$ value \\
\hline Gender (M/F) & & $42 / 11$ & $31 / 9$ & 0.839 \\
\hline Age (year) & & $64.8(10.7)$ & $64.3(12.1)$ & 0.834 \\
\hline \multirow[t]{2}{*}{ Tumor size $(<3 \mathrm{~cm} />3 \mathrm{~cm})$} & $<3 \mathrm{~cm}$ & $40(75 \%)$ & $28(70 \%)$ & 0.556 \\
\hline & $>3 \mathrm{~cm}$ & $13(25 \%)$ & $12(30 \%)$ & \\
\hline \multirow[t]{2}{*}{ Tumor number } & Solitary & $28(53 \%)$ & $25(62 \%)$ & 0.351 \\
\hline & Multiple & $25(47 \%)$ & $15(37 \%)$ & \\
\hline \multirow[t]{2}{*}{ Grade (Low/high) } & Low & $32(60 \%)$ & $15(37 \%)$ & 0.029 \\
\hline & High & $21(40 \%)$ & $25(62 \%)$ & \\
\hline Follow up time (month) & & $83.4(61.7)$ & $72.7(46.7)$ & 0.359 \\
\hline Recurrence (n) & & $29(55 \%)$ & $31(77 \%)$ & 0.023 \\
\hline Progression (n) & & $8(15 \%)$ & $10(25 \%)$ & 0.231 \\
\hline Time to recurrence(month) & & $17.3(29.1)$ & $13.2(14.2)$ & 0.491 \\
\hline Time to progression(month) & & $42.9(49.8)$ & $30.5(38.4)$ & 0.440 \\
\hline
\end{tabular}

according to the tumor invasion regarding the MM-VP: $\mathrm{Tla}$ (invasion above MM-VP), Tlb (MM-VP invasion) bladder cancer 12 . Whenever muscularis mucosae-vascular plexus were not identified the presence of large blood vessels in the upper one-half of the lamina propria coursing parallel to the mucosa was used as a morphologic landmark of the level of the MM (Figure 1) (12).

Statistical analyses were performed using the SPSS software version 20. The Chi-square test and Student t-test were used to compare in two groups. A p value of less than 0.05 was considered to show statistically significant results. For the multivariate analyses, the possible factors identified with univariate analyses were further entered into the logistic regression analyses to determine independent predictor of tumor recurrence.

Hosmer-Lemeshow goodness of fit statistics was used to access model fit. Survival rate was analyzed using the Kaplan-Meier method and compared between the 2 groups with the log-rank test.

\section{RESULTS}

In total 73 male and 20 female patients were included in the study. Mean age at initial diagnosis was 64.6 $(+/-11.3)$ years and mean follow up time was $78.8(+/-$ 58.4) months. Overall, radical cystectomy was performed in 14 patients (15.0\%), and a total of 4 patients $(4.3 \%)$ refused radical cystectomy due to surgical and/or risks of the anesthesia. These patients received radiotherapy plus chemotherapy due to the progression to muscle-invasive disease during this follow-up. Mean recurrence time was $15.2 \pm 22.5$ months and mean pro-

\section{Table 2.}

Logistic regression analysis to determine the independent predictors of recurrence.

\begin{tabular}{|lcccc|}
\hline Risk factors & \multirow{2}{*}{ Sig. } & \multirow{2}{*}{ Exp (B) } & \multicolumn{2}{c|}{ 95\% C.I. for EXP (B) } \\
& & & Lower & Upper \\
\hline Tumor number (solitary vs multiple) & 0.068 & 3.117 & 0.918 & 10.585 \\
\hline Tumor grade (Low vs high ) & 0.000 & 12.643 & 3.680 & 43.432 \\
\hline Substage (T1a vs T1b) & 0.026 & 4.219 & 1.191 & 14.947 \\
\hline Tumor size (<3 cm vs > 3 cm) & 0.006 & 7.325 & 1.758 & 30.525 \\
\hline
\end{tabular}


gression time was $37.1 \pm 44.5$ months considering the all pTl cases.

The results of the comparison of the sub-staging system are shown in Table 1 . There were no statistically significant differences between the two groups according to age, gender, size, multiplicity, follow up time. The MMVP invasion was not detected in 53 tumors $(56.9 \%)$ and patients were classified as pTla. The MM-VP invasion was present in 40 patients (43.0\%) that we classified as pTlb. 25 (47.1\%) for Tla vs. 15(37.5\%) for Tlb had multiple tumors $(p=0.351)$ (Table-1). $40 \%$ and $62 \%$ of patients were high grade BUC in Tla and Tlb, respectively. There was a statistically significant difference between sub-staging according to pTla/pTlb and the WHO 2004 grade system $(\mathrm{p}=0.003)$.

In $29(54.7 \%)$ of pTla and in $31(77.5 \%)$ of $\mathrm{pTlb}$ tumors, the recurrent disease was recorded during the follow-up period. This difference was statistically significant $(\mathrm{p}=0.023)$. In total, 18 patients $(19.3 \%)$ progressed to further stages, and 12 patients (14\%) died of BUC. There was no statistically significant difference between the two groups based on progression (p: 0.231). Stepwise multivariate regression analysis revealed that

\section{Figure 2.}

Kaplan-Meier estimates of disease spesific survival according to invasion of MM-VP in primary T1 transitional cell carcinoma (TCC) of the bladder.

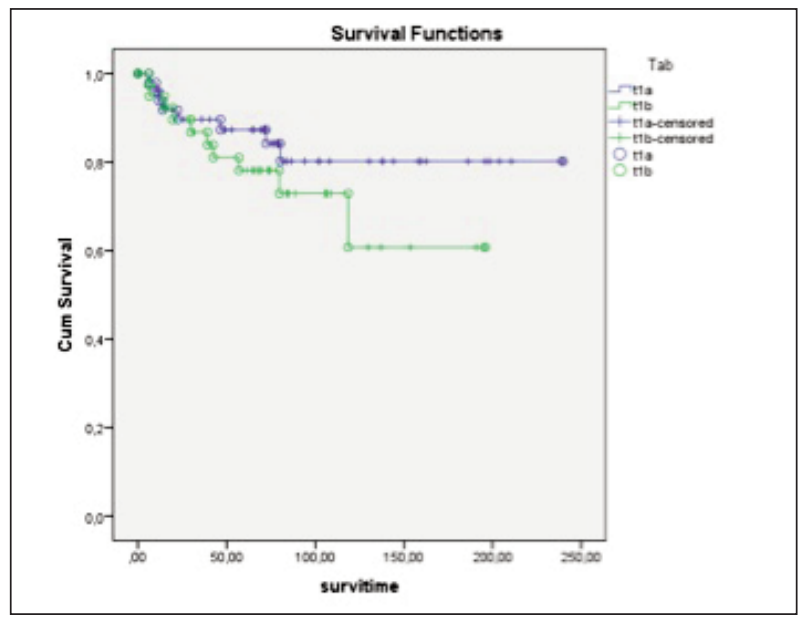

the grade of bladder cancer, the pathological sub-staging system according to the tumor invasion regarding the $\mathrm{MM}-\mathrm{VP}$, and tumor size were the prominent factors affecting the recurrence of bladder cancer (Table 2). Mean recurrence and progression time based on pTla/ pTlb was $17.3 \pm 29.1 / 42.9 \pm 49.8$ months and $13.25 \pm$ $14.2 / 30.5 \pm 38.4$ months, respectively. Although mean recurrence time and progression time longer in pTla group than $\mathrm{pT} 1 \mathrm{~b}$ group, it did not reach statistically significant differences between groups because of the small sample size ( $p>0.05)$. Disease-specific survival (DSS) rates at 5 year for pTla and pTlb were $80.2 \%$ and $60.8 \%$, respectively. Progression free survival (PFS), recurrence free survival (RFS) and DSS rates were similar for $\mathrm{pTla} / \mathrm{pTlb}$ and did not reached statistically significance ( $p>0.05)$ (Figures 2-3).

\section{Discussion}

In this study, sub-staging based on the invasion of MMVP has no effect on progression and recurrence-free survival rates in patients with pTl BUC. However, patients with $\mathrm{pTlb}$ have experienced a higher rate of recurrence during follow-up compared to pTla group. Disease-specific survival was relatively longer in Tla however there was no statistically significant difference in Kaplan-Meier survival estimate analyses.

The management of $\mathrm{Tl}$ bladder cancer is a great challenge for urologists because of its wide and unpredictable range of clinical behavior. Therefore, $\mathrm{pTl}$ disease represents a spectrum of different patients with different tumor behavior. Because of that, investigations have been focused on how to classify and predict the prognosis. Tumor grade, size, multiplicity, the presence of CIS, Re-TUR, lymphovascular invasion, BCG treatment, age, histotype and histological variants, tumor growth pattern, $3^{\text {rd }}$-month cystoscopy results, time to relapse are known parameters that affect prognosis (16). According to TNM classification, pTl tumors recurrence and progression are not homogeneous some of them very aggressive that require early radical cystectomy. Remaining cases should need to follow up with cystoscopies after intravesical therapy. In the contrary, some patients who are treated more aggressively, in fact, may receive overtreatment. However, some

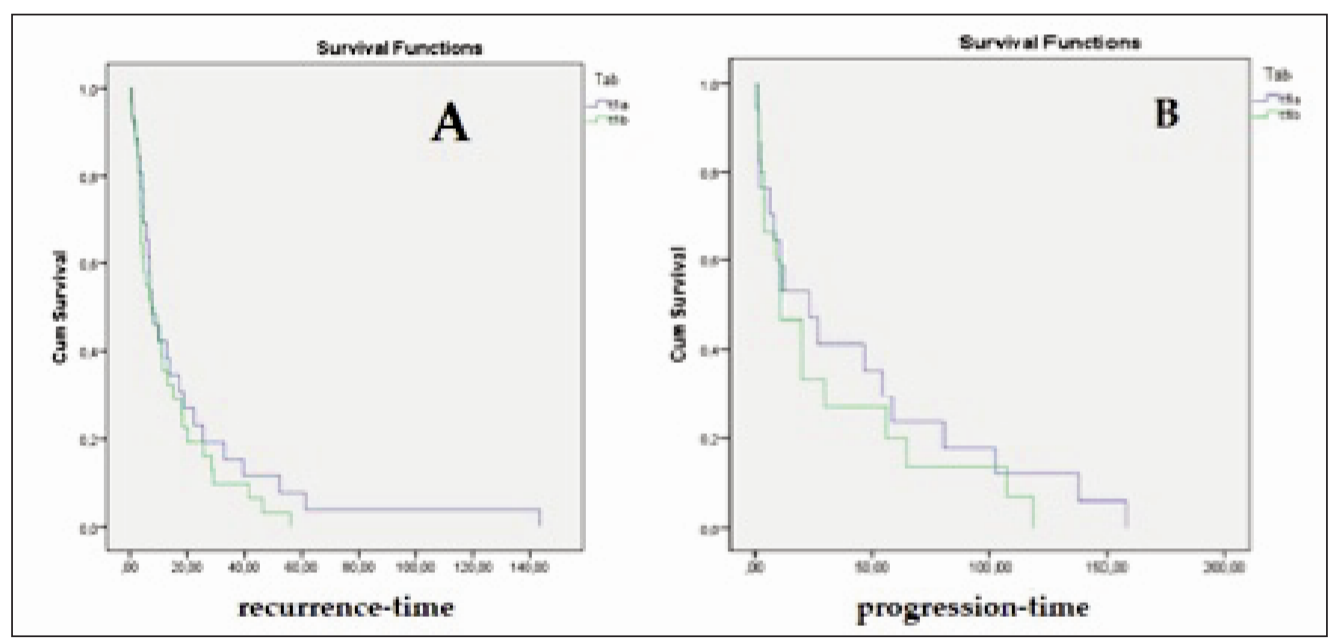

Figure 3.

Kaplan-Meier estimates of recurrence-free $(A)$ and progression-free (B) survival according to invasion of $\mathrm{MM}-\mathrm{VP}$ in primary $\mathrm{T} 1$ transitional cell carcinoma (TCC) of the bladder. Whereas the recurrence-free interval and progression-free were similar for both groups. 
pTl cases may progress to the inoperable stage under the conservative approach. So any improvement to the treatment strategy for the management of pTl BUC is significant. This study proposes that invasion of MM-VP (pTlb) may be associated with a higher and earlier recurrence, although a statistical difference was not shown. Larger series with longer follow-up may show a remarkable distinction for pTlb sub-staging.

Different sub-staging systems have been studied in the literature recently $(11,13,18)$. WHO (2004)/ISUP and clinical guidelines do not recommend the sub-staging of T1 bladder cancer yet $(15,16)$. Invasion of muscularis mucosae-vascular plexus invasion and depth and area to lamina propria invasion were most commonly applied sub-classification $(12,13)$. Orsola et al. substaged according to invasion superficial to, into or beyond the muscularis mucosae $(13,18)$. Holmang et al. substaged based on the absent or presence of MM-VP invasion (Tla/Tlb) as in our study (21). Van Rhijn et al. investigated two substaging systems based on the extent of lamina propria involvement [Tl-microinvasive (Tlm) versus T1-extensive-invasive (Tle)] and invasion to MM-VP 12.

Amin et al. commented that the efficacy of the sub-staging of $\mathrm{Tl}$ bladder tumors is still controversial since lack of consensus to define the depth of invasion criteria and established clinical significance (22). The main problem may be the detection of MM-VP since it is not a constant layer, $6 \%$ to $75 \%$ of pathology specimen were not identified (23). Main studies of interest of MM invasion in T1 NMIBC with staging system and assessment rate were changes between $63 \%$ to $100 \%$ (22). In our study we planned to reclassify the $\mathrm{Tl}$ tumors based on presence or absence of MM-VP invasion and depth invasion to lamina propria: Tla (the tumor does not infiltrate the MM$\mathrm{VP}$ ) and Tlb (the tumor infiltrates and/or invades the [MM-VP]), and T1m (micro-invasive- a single focus of lamina propria invasion with a maximum diameter of $0.5 \mathrm{~mm}$ ) and Tle (extensive-invasive, $>0.5 \mathrm{~mm}$ ). If the MM-VP was not seen at the invasion front, we classify pTla or pTlb according to the depth of invasion into the lamina propria by looking at the MM-VP in tumor-free areas in the same or other TUR slides (12). We classified as $\mathrm{Tla} / \mathrm{b}$ all the patients and classification according to lamina propria invasion depth $(\mathrm{T} 1 \mathrm{~m} / \mathrm{e})<0.5 \mathrm{~mm}$ or $>0.5 \mathrm{~mm}$ was not feasible result in our study. Since only 6 of the patients classified as T1m BCa and all the others reported as TleBCa so we could able to analyze only the presence or absence of MM-VP invasion (Tla/Tlb).

In literature the largest study was reported by Rouprêt et al. with 587 patients, that $\mathrm{pTla} / \mathrm{b}$ sub-staging based on the MM-VP invasion was very predictive of Tl NMIBC behavior as recurrence-free $(\mathrm{p}=0.03)$ progression-free $(p<0.001)$ and cancer-specific survival $(p=0.02)$ in 35 months median follow up time (24). In our study, pTla BUC a was a higher recurrence rate of $29(54.7 \%)$ than Tlb 31(77.5\%) (p: 0.023) but there were no statistically significant differences between two groups with Kaplan Meier analyses (log rank, p-value > 0.05) in 78.8 (58.4) months mean follow-up time.

Skoup et al. reported that Tl sub-staging was the indepented prognostic factors for tumour progression ( $\mathrm{p}<$ $0.0001)$, cancer-specific survival $(p=0.0001)$ and over- all survival $(p=0.0002)$ (25). De-Marko et al. analyzed two sub-staging systems for Tl bladder cancers based on $\mathrm{MM}-\mathrm{VP}$ invasion (Tla/b/c) and lamina propria invasion depth $(\mathrm{T} 1 \mathrm{~m} / \mathrm{e})$ two sub-staging system were not reached prognostic significance level for progression-free survival and disease-specific survival after 9.5 years of follow-up 20. Van Rhijn et al. evaluated MM-VP invasion depth as $\mathrm{Tla} / \mathrm{Tlb} / \mathrm{Tlc}$ and lamina propria invasion above or below to $0.5 \mathrm{~mm}(\mathrm{Tlm} / \mathrm{Tle})$, which show a higher predictive value for disease progression and disease-specific survival (12). Orsola et al. reported that sub-staging using depth of lamina propria invasion was significant for progression (13). In our study, there were no statistically significant differences between disease progression, recurrence and cancer-specific survival between the Tla/b sub-staging systems.

Patriarca et al. report that $1 \mathrm{~mm}$ invasion system predicted progression $(\mathrm{p}<0.04)$ and Re-TUR increase the survival rate (26). They reclassified $1 \mathrm{~mm}$ sub-staging system in $100 \%$ of cases, the $11 \mathrm{~m} / \mathrm{e}$ in $100 \%$, and the anatomy-based method (T1 a/b) in $72.3 \%$ of cases (26). In our study, we detected only 6 cases with Tlm groups based on $0.5 \mathrm{~mm}$ threshold, so $1 \mathrm{~mm}$ of invasion threshold might be more useful results clinically. Finally, although the EORTC and CUETO risk scores improve risk stratification by quantifying recurrence and progression possibilities, their performance remains imperfect. These scoring systems may further improve with using this sub-staging system (27).

Our study has some limitations such as reporting the retrospective data of a relatively small patient group. Our results could not show statistically significant differences according to disease-specific survival and progressionfree survival because of the small sample size.

\section{Conclusions}

Based on the findings presented in this study, sub-staging of T1 BUC according to the muscularis mucosae-vascular plexus invasion showed a limited impact on the outcome in our patient cohort. However, the presence of pTlb disease was found to be associated with significantly higher of recurrence.

\section{REFERENCES}

1. van Rhijn BW, Burger M, Lotan Y, et al. Recurrence and progression of disease in non-muscle-invasive bladder cancer: from epidemiology to treatment strategy. Eur Urol. 2009; 56:430-42.

2. Burger M, Oosterlinck W, Konety B, et al. ICUD-EAU International Consultation on Bladder Cancer 2012: Non-muscle-invasive urothelial carcinoma of the bladder. Eur Urol. 2013; 63:36-44.

3. Hall MC, Chang SS, Dalbagni G, et al. Guideline for the management of nonmuscle invasive bladder cancer (stages Ta, T1, and Tis): 2007 update. J Urol. 2007; 178:2314-30.

4. Kulkarni GS, Hakenberg OW, Gschwend JE, et al. An updated critical analysis of the treatment strategy for newly diagnosed high-grade T1 (previously T1G3) bladder cancer. Eur Urol. 2010; 57:60 70.

5. Cambier S, Sylvester RJ, Collette L, et al. EORTC Nomograms and Risk Groups for Predicting Recurrence, Progression, and Disease-specific and Overall Survival in Non-Muscle-invasive Stage 
Ta-T1 Urothelial Bladder Cancer Patients Treated with 1-3 Years of Maintenance Bacillus Calmette-Guerin. Eur Urol. 2016; 69:60-9.

6. Özkaptan O, Çubuk A, Dinçer E, et al. Extraperitoneal antegrade vs transperitoneal open radical cystectomy: single center experiences with 200 cases. Bladder Cancer 2020; 6:187-194.

7. Budak S, Yucel C, Keskin MZ, et al. Pathology outcomes in patients with transurethral bladder tumour resection in a Turkish population: A retrospective analysis. Arch Ital Urol Androl. 2018; 90:8-10.

8. Mathieu R, Lucca I, Roupret M, et al. The prognostic role of lymphovascular invasion in urothelial carcinoma of the bladder. Nat Rev Urol. 2016; 13:471-9.

9. Breyer J, Bertz S, Müller A, et al. New pathological features predicting prognosis of early-invasive urothelial carcinoma: Quantitative substaging and tumour invasion pattern should assist WHO 1973 grading classification in predicting cancer-specific survival of stage pT1 bladder cance. Eur Urol. Suppl 2016;15:e392.

10. Naselli A, Hurle R, Paparella S, et al. Role of restaging transurethral resection for $T 1$ non-muscle invasive bladder cancer: a systematic review and meta-analysis. Eur Urol Focus. 2018; 4:558-567.

11. Younes M, Sussman J, True LD. The usefulness of the level of the muscularis mucosae in the staging of invasive transitional cell carcinoma of the urinary bladder. Cancer. 1990; 66:543-8.

12. van Rhijn BW, van der Kwast TH, Alkhateeb SS, et al. A new and highly prognostic system to discern $T 1$ bladder cancer substage. Eur Urol. 2012; 61:378-84

13. Orsola A, Trias I, Raventos CX, et al. Initial high-grade TI urothelial cell carcinoma: feasibility and prognostic significance of lamina propria invasion microstaging $(T 1 \mathrm{a} / \mathrm{b} / \mathrm{c})$ in BCG-treated and BCG-non-treated patients. Eur Urol. 2005; 48:231-8.

14. Turan T, Efiloglu O, Gunaydin B, et al. Comparative differences between $\mathrm{Tl} \mathrm{a} / \mathrm{b}$ and $\mathrm{T} 1 \mathrm{e} / \mathrm{m}$ as substages in $\mathrm{T} 1$ urothelial carcinoma of the bladder. Int Braz J Urol. 2018; 44:267-72.

15. Babjuk M, Bohle A, Burger M, et al. EAU Guidelines on nonmuscle-invasive urothelial carcinoma of the bladder: update 2016. Eur Urol. 2017; 71:447-61.

16. Humphrey PA, Moch H, Cubilla AL, et al. The 2016 WHO clas- sification of tumours of the urinary system and male genital organsPart B: prostate and bladder tumours. Eur Urol. 2016; 70:106-19.

17. Smits G, Schaafsma E, Kiemeney L, et al. Microstaging of pT1 transitional cell carcinoma of the bladder: identification of subgroups with distinct risks of progression. Urology. 1998; 52:1009-13

18. Lee JY, Joo HJ, Cho DS, et al. Prognostic significance of substaging according to the depth of lamina propria invasion in primary $\mathrm{TI}$ transitional cell carcinoma of the bladder. Korean J Urol. 2012; $53: 317-23$

19. Platz CE, Cohen MB, Jones MP, et al. Is microstaging of early invasive cancer of the urinary bladder possible or useful? Mod Pathol. 1996; 9:1035-9.

20. De Marco V, Cerruto MA, D'Elia C, et al. Prognostic role of substaging in T1G3 transitional cell carcinoma of the urinary bladder. Mol Clin Oncol. 2014; 2:575-80.

21. Holmang $S$, Hedelin $H$, Anderstrom $C$, et al. The importance of the depth of invasion in stage T1 bladder carcinoma: a prospective cohort study. J Urol. 1997; 157:800-3

22. Amin MB, McKenney JK, Paner GP, et al. ICUD-EAU International Consultation on Bladder Cancer 2012: Pathology. Eur Urol. 2013; 63:16-35

23. Ro JY, Ayala AG, el-Naggar A. Muscularis mucosa of urinary bladder. Importance for staging and treatment. Am J Surg Pathol. 1987; 11:668-73

24. Roupret M, Seisen T, Comperat E, et al. Prognostic interest in discriminating muscularis mucosa invasion (Tla vs Tlb) in nonmuscle invasive bladder carcinoma: French national multicenter study with central pathology review. J Urol. 2013; 189:2069-76.

25. Soukup V, Duskova J, Pesl M, et al. The prognostic value of T1 bladder cancer substaging: a single institution retrospective study. Urol Int. 2014; 92:150-6

26. Patriarca $C$, Hurle $R$, Moschini $M$, et al. Usefulness of $p T 1$ substaging in papillary urothelial bladder carcinoma. Diagn Pathol. 2016; 11:6.

27. Gershman B, Boorian SA, Hautmann RE. Management of TI urothelial carcinoma of the bladder: what do we know and what do we need to know? Bladder Cancer 2015; 2:1-14.

\author{
Correspondence \\ Ahmet Sahan, MD \\ Alkan Cubuk, MD (Corresponding Author) \\ alkancubuk@hotmail.com \\ Orkunt Ozkaptan, MD \\ Kartal Dr. Lutfi Kirdar Training and Research Hospital, Department of Urology, \\ Cevizli Mh S.emsi Denizer Cad. E-5 Karayolu Cevizli Mevkii, 34890 Kartal \\ Istanbul (Turkey) \\ Fatma Gerin, MD \\ Emine Bozkurtlar, MD \\ Marmara University, Department of Pathology, Istanbul (Turkey) \\ Asgar Garayev, MD \\ Yiloren Tanidir, MD \\ Haydar Kamil Cam, MD \\ Ilker TinaY, MD \\ Marmara University, Department of Urology, İstanbul (Turkey) \\ Kastm Ertas, MD \\ Yuzuncu Yll University, Department of Urology, Van (Turkey)
}

